\title{
Influence of Impulse Disturbances on Oscillations of Nonlinearly Elastic Bodies
}

\author{
Andriy Andrukhiv ${ }^{1} \mathbb{D}$, Mariia Sokil $^{2}$, Bohdan Sokil $^{3}$, Solomiia Fedushko ${ }^{4, *} \mathbb{C}$, Yuriy Syerov ${ }^{4, * \mathbb{D}}$, \\ Vincent Karovic, Jr. ${ }^{5}$ and Tetiana Klynina ${ }^{6}$
}

1 Scientific Library, Lviv Polytechnic National University, 79007 Lviv, Ukraine; andriy.i.andrukhiv@lpnu.ua

2 Department of Transport Technologies, Institute of Engineering Mechanics and Transport, Lviv Polytechnic National University, 79007 Lviv, Ukraine; mariia.b.sokil@lpnu.ua

3 Department of Engineering Mechanics, Hetman Petro Sahaidachnyi National Army Academy, 79012 Lviv, Ukraine; sokil_b_i@ukr.net

4 Department of Social Communication and Information Activities, Institute of the Humanities and Social Sciences, Lviv Polytechnic National University, 79007 Lviv, Ukraine

5 Department of Information Systems, Faculty of Management, Comenius University in Bratislava, 82005 Bratislava, Slovakia; vincent.karovic2@fm.uniba.sk

6 History and Documentation Department, National Aviation University, 03058 Kyiv, Ukraine; tklynina@gmail.com

* Correspondence: solomiia.s.fedushko@lpnu.ua (S.F.); yurii.o.sierov@lpnu.ua (Y.S.)

check for updates

Citation: Andrukhiv, A.; Sokil, M.; Sokil, B.; Fedushko, S.; Syerov, Y.; Karovic, V., Jr.; Klynina, T. Influence of Impulse Disturbances on Oscillations of Nonlinearly Elastic Bodies. Mathematics 2021, 9, 819. https: / / doi.org/10.3390/math9080819

Academic Editor: Natalia Kryvinska

Received: 15 February 2021

Accepted: 1 April 2021

Published: 9 April 2021

Publisher's Note: MDPI stays neutral with regard to jurisdictional claims in published maps and institutional affiliations.

Copyright: (c) 2021 by the authors. Licensee MDPI, Basel, Switzerland. This article is an open access article distributed under the terms and conditions of the Creative Commons Attribution (CC BY) license (https:/ / creativecommons.org/licenses/by/ $4.0 /)$.
Abstract: A method for studying the effect of impulse perturbation on the longitudinal oscillations of a homogeneous constant cross-section of the body and the elastic properties of a material which satisfies the essentially nonlinear law of elasticity has been developed. A mathematical model of the process is presented, which is an equation of hyperbolic type with a small parameter at the discrete right-hand side. The latter expresses the effect of impulse perturbation on the oscillatory process. As for the boundary conditions considered in the work, they are classic of the first, second and third genera. The methodology is based on: the principle of oscillation frequency in nonlinear systems with many degrees of freedom and distributed parameters; basic provisions of asymptotic methods of nonlinear mechanics; the idea of using special periodic Ateb-functions to construct solutions of some classes of nonlinear differential equations; properties of completeness and orthonormality of functions that describe the forms of oscillations of undisturbed motion. In general, the above allowed to obtain relations that describe for the first approximation the defining parameters of the oscillations of an elastic body. Their peculiarity is that even for undisturbed motion, the natural frequency of oscillations depends on the amplitude, and therefore, under the action of a periodic (over time) pulse force on the elastic body, both resonant and nonresonant processes are possible in the latter. It, in contrast to an elastic body with linear or quasilinear elastic properties of the body is determined not only by its basic physical and mechanical properties, but also by the amplitude of oscillations. As a special case, the oscillations of the body under the action of a constant periodic momentum perturbation are considered. It is shown that for the nonresonant case for the first approximation it does not affect the laws of change of amplitude and frequency of the process. As for the resonant is the amplitude of origin through the main resonance significantly depends not only on the speed but also on the points of action of the pulsed perturbation. Moreover, the closer the point of application of the pulsed force to the middle of the elastic body under boundary conditions of the first kind is greater (for boundary conditions of the second kind closer to the end).

Keywords: nonlinear oscillations; pulse perturbation; single-frequency oscillatory process

\section{Introduction}

Oscillatory processes of one-dimensional models of elastic bodies (longitudinal, torsional, bending) elastic properties of which are described by linear or close to them relations were considered, as a rule, for the case of continuous action of external factors on them. 
The most important of them are cases of their periodic action. Periodic action (even at a small value) under certain conditions can lead to so-called resonant phenomena in elastic bodies. These phenomena are characterized by a significant increase in the amplitudes of oscillations, and thus - an increase in dynamic loads, which leads to a decrease in the service life of the elastic body and the node or mechanism of which they are part. Therefore, to predict them and describe the features of the passage is an important issue. However, linear or quasilinear relations do not always accurately describe the real process of their deformations (especially for their significant quantities), in addition, there are a number of body materials whose elastic properties are described by nonlinear relations [1]. They can be described by a ratio with a sufficient degree of accuracy $\sigma=E \varepsilon^{v+1}+\mu f(\varepsilon, \dot{\varepsilon})$, where respectively $\sigma, E, \varepsilon$ stress, "modulus of elasticity", the relative deformation of the material of the protective element, $\mu f(\varepsilon, \dot{\varepsilon})$ is analytical function that describes the small deviation of the elastic properties of the material from the power law why indicates a small parameter $\mu, v+1=\frac{2 m+1}{2 n+1}, m, n=0,1,2, \ldots$.

Dynamic processes in such elastic bodies [2-6] require the consideration of qualitatively new mathematical models $[7,8]$, and, consequently, the development of new or generalization of existing analytical methods for their research [9-12].

In part, they have been developed for one-dimensional models of elastic bodies or media under continuous perturbation [13-15]. As for the study of processes in such bodies for the case of instantaneous (pulse) or random force action, they have not found proper development due to purely mathematical problems [16]. It is the development of methods for studying the longitudinal oscillations of the body whose elastic properties are described as close to the power law of elasticity under the action of impulse perturbation on it is the subject of consideration.

The main aim of this investigation is to study the influence of impulse disturbances on oscillations of nonlinearly elastic bodies.

A technique for studying the effect of impulse perturbation on the longitudinal oscillations of substantially nonlinearly elastic bodies will be developed in this study.

Using this technique, we will analyze the relationship of the studied process in linear (quasilinear) models of elastic bodies and essentially nonlinear models.

\section{Mathematical Model of Longitudinal Oscillations of an Elastic Body under the Impulse Action of External Perturbation}

Mathematical model of longitudinal oscillations of a homogeneous body, the material of which satisfies close to the power law of elasticity under the action on it at certain points of small-magnitude impulse perturbation, serves at certain marginal differential equations.

$$
u_{t t}-\alpha^{2}\left(u_{x}\right)^{v} u_{x x}=\frac{\mu}{\rho}\left(f\left(u, u_{t}, u_{x}, u_{x x}\right)+\sum_{s=1} g_{s}\left(u, u_{t}, u_{x}, u_{x x}\right) \delta\left(t-t_{s}\right) \delta\left(x-x_{s}\right)\right),
$$

in which $u(x, t)$ is the movement of the cross section of the body with the coordinate $x$ at any time $t, f\left(u, u_{t}, u_{x}, u_{x x}\right)$ is a function that describes the deviation of the elastic characteristics of the body from the power law and the force of viscoelastic friction, the maximum value of which is a small value compared to the maximum value of the elastic force (internal) $E A\left(u_{x}\right)^{v} u_{x x}\left(A\right.$ is a cross-sectional area of the body, $\alpha^{2}=\frac{E A}{m}, m$ is a running mass of elastic body material), $\mu$ is a small parameter, $g_{s}\left(u, u_{t}, u_{x}, u_{x x}\right) \delta\left(t-t_{s}\right) \delta\left(x-x_{s}\right)$ is a function that describes the magnitude of the momentum perturbation acting on an elastic body at a point with a coordinate $x_{s}$ at time $t_{s}$. Here and below, the parameter $v$ satisfies the condition $v+1=\frac{2 m+1}{2 n+1}, m, n=0,1,2, \ldots$, that ensures the existence of a periodic solution in the undisturbed $(\mu=0)$ equation which corresponds to Equation (1).

It should be noted that the question of the existence of a periodic solution in nonlinear differential equations corresponding to the undisturbed Equation (1) was considered, for example [17], where it is shown that its period on the time variable depends on the initial conditions, and in [18] its asymptotic is constructed without taking into account the impulse action, i.e., in the case $g_{s}\left(u, u_{t}, u_{x}, u_{x x}\right) \equiv 0$. 
The most interesting and at the same time the most important from the practical point of view is the case when the time between the next two actions of impulse perturbation is constant: $t_{s+1}-t_{s}=\tau$, that is, the impulse perturbation is periodic.

This is the case we will consider below. For this case, the effect of impulse perturbation on the body is somewhat converted.

The transformation is based on the basic properties $\delta$ functions [19-21] and the frequency of impulse perturbation:

(a) from $\tau$ the periodicity of the perturbation follows

$$
\sum_{s=1} g_{s}\left(u, u_{t}, u_{x}, u_{x x}\right) \delta\left(t-t_{s}\right) \delta\left(x-x_{s}\right)=\sum_{s=1} g_{s}\left(u, u_{t}, u_{x}, u_{x x}\right) \delta\left(x-x_{s}\right) \delta(t-(s-1) \tau)
$$

(b) from the main properties of the $\delta$ function [22] it follows that with a sufficient degree of accuracy the pulse component of the external perturbation can be represented as

$$
\begin{gathered}
\sum_{s=1} g_{s}\left(u, u_{t}, u_{x}, u_{x x}\right) \delta\left(x-x_{s}\right) \delta(t-2(s-1) \tau)=\sum_{s=1} g_{s}\left(u, u_{t}, u_{x}, u_{x x}\right) \delta\left(x-x_{s}\right) \\
\cos \theta \delta\left(\frac{\theta}{\Omega}-\frac{2(s-1) \pi}{\Omega}\right) \\
=\sum_{s=1} g_{s}\left(u, u_{t}, u_{x}, u_{x x}\right) \delta\left(x-x_{s}\right) \cos \theta \delta\left(\frac{\theta}{\Omega}-\frac{2(s-1) \pi}{\Omega}\right)
\end{gathered}
$$

where $\theta=\Omega t, \Omega=\frac{2 \pi}{\tau}$ and the lower parameter $\theta$ will be called the phase of external periodic perturbation.

As for the boundary conditions for Equation (1), they are classically homogeneous

$$
\begin{gathered}
u(x, t)_{\mid x=0}=0, u(x, t)_{\mid x=l}=0, \\
u(x, t)_{\mid x=0}=0, u(x, t)_{\mid x=l}=0, \\
u(x, t)_{\mid x=0}=0, u_{x}(x, t)_{\mid x=l}=0,
\end{gathered}
$$

and correspond to the fixed Equation (4), free Equation (5) and fixed left and free right end Equation (6) of the elastic rectilinear body.

Thus, the problem of the influence of periodic impulse perturbation on a dynamic process is reduced to the construction and study of the solution of a differential equation

$$
\begin{gathered}
\frac{\mu}{\rho}\left(f\left(u, u_{t}, u_{x}, u_{x x}\right)+\sum_{s=1} g_{s}\left(u, u_{t}, u_{x}, u_{x x}\right) \delta\left(x-x_{s}\right) \cos \theta \delta\left(\frac{\theta}{\Omega}-\frac{2(s-1) \pi}{\Omega}\right)\right),
\end{gathered}
$$

under boundary Conditions Equation (4), Equation (5) or Equation (6).

\section{Method of Constructing an Asymptotic Approximation of the Solution of a Mathematical Model of Nonlinear Oscillations of Elastic Body under Pulsed Action of Forces}

For him, the first approximation of the asymptotic solution in the form close to one of the forms of dynamic equilibrium [14,23] is presented in the form

$$
\begin{aligned}
& u_{k}(x, t)=a_{k}(t) X_{k}(x) c a\left(v+1,1, \psi_{k}(t)\right)+\varepsilon U_{1 k}\left(a_{k}, x, \psi_{k}, \theta\right) \\
& \psi_{k}(t)=\omega_{k}\left(a_{k}\right) t+\vartheta_{k},
\end{aligned}
$$

where $a_{k}(t)$ and $\psi(t)_{k}$ are parameters that together with the function $U_{1 k}\left(a_{k}, x, \psi_{k}, \theta\right)$ take into account the influence of the right-hand side of Equation (7) on the oscillatory process of an elastic body; $\omega_{k}\left(a_{k}\right)=\alpha\left(\frac{\lambda_{k} \Pi_{x}}{l}\right)^{1+\frac{v}{2}} a_{k}{ }^{\frac{v}{2}}$ and $X_{k}(x)$ is an accordingly, the frequency of natural oscillations (therefore, the frequency of natural oscillations $\mu=0$ ) and own 
functions. The latter describe the forms of oscillations and are expressed for the considered boundary conditions through periodic Ateb- functions [23-25] by dependences (Figure 1).

$$
\left\{X_{k}(x)\right\}=\left\{\begin{array}{c}
s a\left(1, \frac{1}{v+1}, \frac{\lambda_{k}}{l} \Pi_{x} x\right), \\
c a\left(1, \frac{1}{v+1} \frac{\lambda_{k}}{l} \Pi_{x} x\right), \\
s a\left(1, \frac{1}{v+1}, \frac{\lambda_{k}}{l} \Pi_{x} x\right)
\end{array}\right\},
$$

in which $\lambda_{k}$ for boundary conditions Equations (4) and (5) takes values $k$ and $\lambda_{k}=$ $\frac{2 k+1}{2}$ is a for boundary condition Equation (6); $\Pi_{x}$ is the half-life of the functions used, $\left.\Pi_{x}=\sqrt{\pi} \Gamma\left(\frac{v+1}{v+2}\right)\left(\Gamma\left(\frac{1}{2}+\frac{v+1}{v+2}\right)\right)^{-1}\right)$.

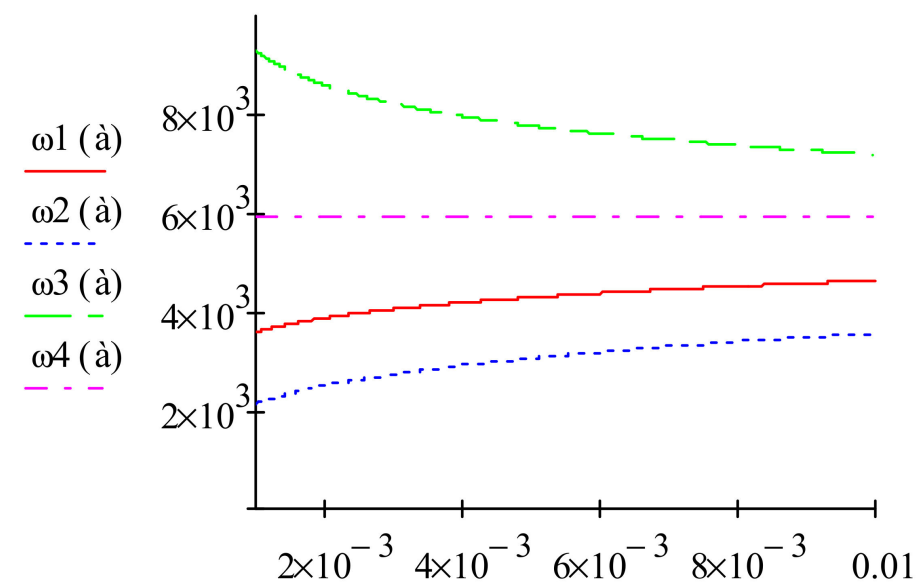

(a)

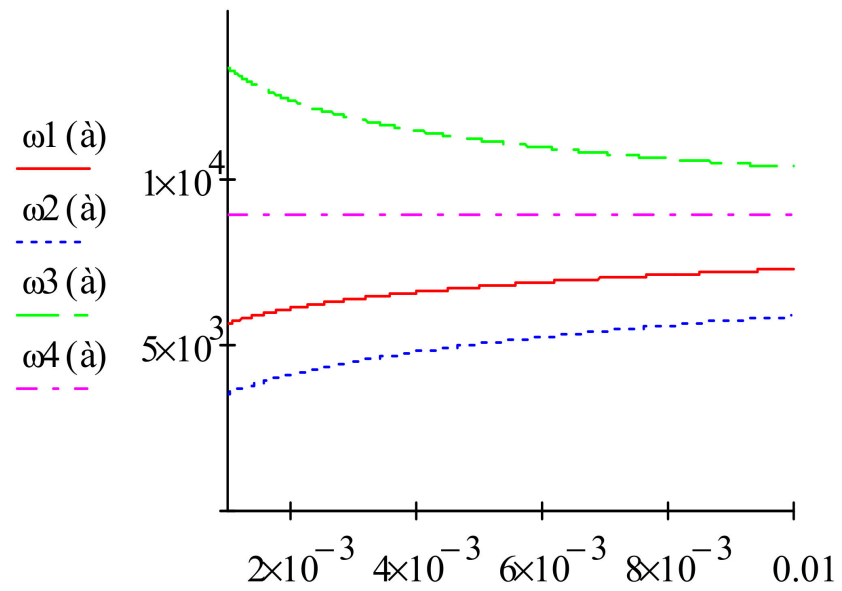

(b)

Figure 1. Dependence of the frequency of natural oscillations of undisturbed motion on the amplitude at different values (a) and $(\mathbf{b})$ of the parameter $v=\frac{2}{9}$ red $v=\frac{4}{9}$ bluev $=0$ orangev $=\frac{-2}{9}$ green, $\mathbf{a}=10^{4}$.

Thus, the peculiarity of the dynamic process of undisturbed motion is its non-isochrony, moreover, for the case $v>0$ a larger value of the amplitude of natural oscillations corresponds to a larger value of natural frequency; for the case $-1<v<0$ there is an inverse relationship.

Regarding the function $U_{1 k}\left(a_{k}, x, \psi_{k}, \theta\right)$ in representation Equation (8), it must be $2 \Pi$ periodic by argument $\psi_{k}$ and $2 \pi$ is periodic by argument $\theta$ and does not contain the first argument modes $\psi_{k}\left(2 \Pi=2 \sqrt{\pi} \Gamma\left(\frac{1}{v+2}\right)\left(\Gamma\left(\frac{1}{2}+\frac{1}{v+2}\right)\right)\right)$. These conditions are equivalent to the choice of the amplitude of the amplitude $k$ is its mode. Thus, it is necessary to 
determine such relations that describe the laws of change of parameters $a_{k}(t)$ and $\psi_{k}(t)$ so that the asymptotic representation of Solution in the Equation (8) satisfies the original Equation (1) or its analogue Equation (7) with the considered accuracy. It should be noted that the system functions $\left\{X_{k}(x)\right\}$, which describes the forms of natural oscillations has the property of completeness and normalization on the interval $[0, l]$, that is:

$$
\int_{0}^{l} X_{p}(x) X_{q}(x) d x=\left\{\begin{array}{l}
\text { Owhen } p \neq q \\
\text { Pwhen } p=q
\end{array},\right.
$$

And $P=\frac{v+2}{3 v+4} l$ for boundary conditions in the Equations (4) and (5) and $P=\frac{v+2}{3 v+4} \frac{l}{2}$ is for boundary condition in the Equation (6);

Without going into mathematical calculations, to obtain a connection between unknown functions we obtain a differential equation:

$$
\begin{aligned}
& \frac{\partial^{2} U}{\partial \psi^{2}} \omega^{2}(a)+\frac{\partial^{2} U}{\partial \theta^{2}} \mu^{2}+2 \frac{\partial^{2} U}{\partial \theta \partial \psi} \mu \omega(a)-\alpha^{2}\left(X^{\prime}(x)\right)^{v} \frac{\partial^{2} U}{\partial x^{2}}= \\
& =\frac{2}{(v+2)}\left[\frac{d a}{d t}\left(\omega(a)+a \frac{d \omega}{d a}\right) s a(1, v+1, \psi)+a \omega(a) \frac{d \psi}{d t} c a^{v+1}(v+1,1, \psi)\right] X(x)+ \\
& +\rho^{-1} F(a, x, \psi)+(\rho P)^{-1} \sum_{s=1} G_{s}(a, x, \psi) \cos \theta \delta\left(\frac{\theta}{\Omega}-\frac{2(s-1) \pi}{\Omega}\right) \sum_{j=1} X_{j}\left(x_{i}\right) X_{j}(x),
\end{aligned}
$$

where $F(a, x, \psi)$ and $G_{s}(a, x, \psi)$ correspond to the values of the functions $f\left(u, u_{t}, u_{x}, u_{x x}\right)$ and $g_{s}\left(u, u_{t}, u_{x}, u_{x x}\right)$ provided they have $u(x, t)$ and its derivatives are determined according to the main value of the specified function, i.e., $u(x, t)=a X(x) c a(v+1,1, \psi)$.

Notes:

1. In the differential Equation (9) and below the indices $k$ which indicates the form of "dynamic equilibrium" of the elastic body for ease of recording omitted;

2. Quantity $\omega(a)+a \frac{d \omega}{d a}$ according to the frequency of natural oscillations takes values $\frac{v+2}{2} \omega(a)$;

3. Depending on the Equation (10) function $\delta\left(x-x_{s}\right)$ based on the properties of the system of functions that describe the forms of oscillations of undisturbed motion are presented in the form $\delta\left(x-x_{s}\right)=P^{-1} \sum_{j=1} X_{j}\left(x_{s}\right) X_{j}(x)$.

\subsection{Amplitude-Frequency Characteristics of Non-Resonant Oscillations of an Elastic Body}

The differential Equation (11) obtained above is the basis for the analytical finding of the laws of change of the amplitude and frequency of oscillations of an elastic body.

If function $U(a, x, \psi, \theta)$ is $2 \Pi$ periodic by argument $\psi$ and does not contain in the schedule of the first harmonic $\psi$, then its derivatives have the same property, and hence, as follows from differential Equation (11), the coefficients for the first harmonics $\psi$ its right part must be equal to zero. This property allows you to find the relationship to determine the laws of change in time of the rate of change of amplitude $\frac{d a}{d t}$ and phase change rate $\frac{d \psi}{d t}$ dynamic process in the form:

$$
\begin{aligned}
& \frac{d a}{d t}=-\frac{\mu s a(1, v+1, \psi)}{2 \omega(a) \rho P} \int_{0}^{l}\left\{F(a, x, \psi)+P^{-1} \sum_{s=1} G_{s}(a, x, \psi) \cos \theta \delta\left(\frac{\theta}{\mu}-\frac{2(s-1) \pi}{\mu}\right) \sum_{j=1} X_{j}\left(x_{s}\right) X_{j}(x)\right\} X(x) d x \\
& \frac{d \psi}{d t}=\omega(a)-\frac{\mu(v+2) c a^{v+1}(v+1,1, \psi)}{2 a \omega(a) \rho P} \int_{0}^{l}\left\{F(a, x, \psi)+P^{-1} \sum_{s=1} G_{s}(a, x, \psi) \cos \theta \delta\left(\frac{\theta}{\mu}-\frac{2(s-1) \pi}{\mu}\right) \sum_{j=1} X_{j}\left(x_{s}\right) X_{j}(x)\right\} X(x) d x
\end{aligned}
$$

From the physical content of the obtained relations and their periodicity according to the arguments, it follows that in the initial system there are two possible cases: nonresonant and resonant. First, consider a simpler nonresonant. It occurs when the period of natural oscillations $\left.\frac{2 \Pi}{\omega(a)}\right)$ not related to the rational relationship with the period of impulse perturbation $\tau$, that is $p \frac{2 \Pi}{\omega(a)} \neq q \tau, p, q$ are mutually prime numbers. For this case, the dynamic 
process is described by somewhat simpler relations, which are formed from Equation (12) by averaging their right-hand parts by the phases of natural and forced oscillations. The basis for performing this procedure is the proportionality of the rate of change of the amplitude to a small parameter, and therefore during the period of oscillations the amplitude changes by a value proportional to the small parameter $\mu$. We get:

$$
\begin{aligned}
& \frac{d a}{d t}=\mu A_{1}(a)-\frac{\mu}{4 \omega(a) \rho P^{2} \Pi} \int_{0}^{2 \Pi} \int_{0}^{l}\left\{\sum_{s=1} \cos (2(s-1) \pi) G_{s}(a, x, \psi) \sum_{j=1} X_{j}\left(x_{s}\right) X_{j}(x)\right\} X(x) s a(1, v+1, \psi) d x d \psi, \\
& \frac{d \psi}{d t}=\omega(a)-\mu B_{1}(a)-\frac{\mu(v+2)}{8 \pi a \omega(a) P^{2} \Pi} \int_{0}^{2 \Pi} \int_{0}^{l}\left\{\sum_{s=1} \cos (2(s-1) \pi) G_{s}(a, x, \psi) \sum_{j=1} X_{j}\left(x_{s}\right) X_{j}(x)\right\} X(x) c a(v+1, \psi) d x d \psi
\end{aligned}
$$

where

$$
\begin{aligned}
& A_{1}(a)=\frac{-1}{4 \omega(a) \rho P \Pi} \int_{0}^{2 \Pi} \int_{0}^{l}\{F(a, x, \psi)\} X(x) s a(1, v+1, \psi) d x d \psi, \\
& B_{1}(a)=-\frac{(v+2)}{4 a \omega(a) P \Pi} \int_{0}^{2 \Pi} \int_{0}^{l}\{F(a, x, \psi)\} X(x) c a(v+1, \psi) d x d \psi .
\end{aligned}
$$

3.2. Resonant Oscillations of a Substantially Nonlinear Elastic Body under the Action of Periodic Impulse Forces

As for the resonant case, which from the theoretical and practical point of view is much more important (Figure 2), for him:

- First, the defining parameters that describe the dynamics of the process significantly depend on the "phase difference" $\gamma=\frac{\pi}{\Pi} \psi-\theta \rightarrow \psi=\frac{\Pi}{\pi}(\gamma+\theta)$ natural oscillations and periodic perturbations;

- Secondly, it takes place under the condition that the amplitude of the process approaches the value $a^{*}=\left(\frac{2 \Pi}{\alpha \tau}\right)^{\frac{2}{v}}\left(\frac{l}{\lambda \Pi_{x}}\right)^{1+\frac{2}{v}}$;

- Third, if the initial amplitude is less than $a^{*}$, and function $f\left(u, u_{t}, u_{x}, u_{x x}\right)$ describes only dissipative and nonlinearly elastic forces, then a small periodic momentum perturbation can cause a resonant process in an elastic body cannot;

- Fourth, the main part of the speed of the "phase difference" $\frac{d \gamma}{d t}$ for the case of transition through resonance, as follows from the above, can be presented as $\frac{\pi}{\Pi} \omega(a)_{\mid a=a^{*}}-\frac{2 \pi}{\tau}$ or $\frac{d \omega(a)}{d a}{ }_{\mid a=a *}\left(a-a^{*}\right)$.

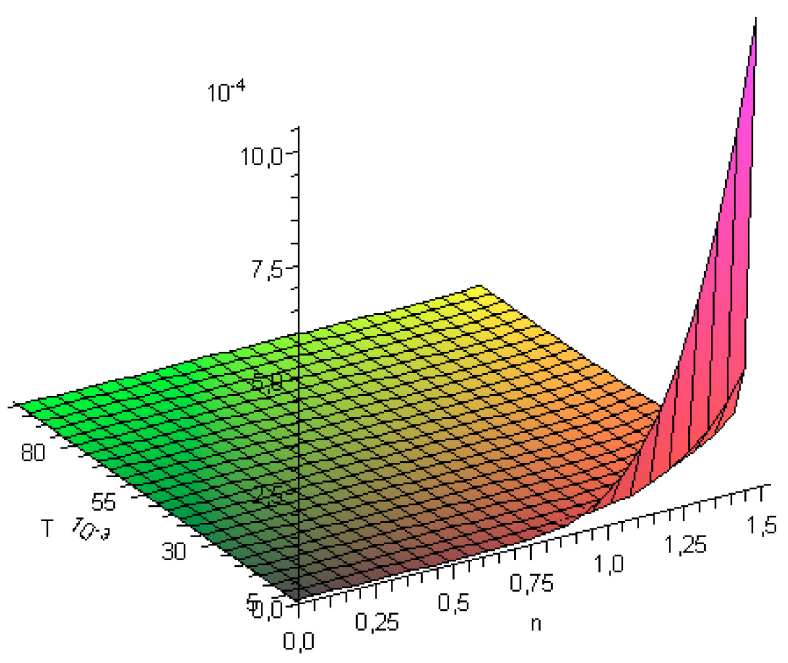

(a) $l=1, \alpha=10^{4}$

Figure 2. Cont. 


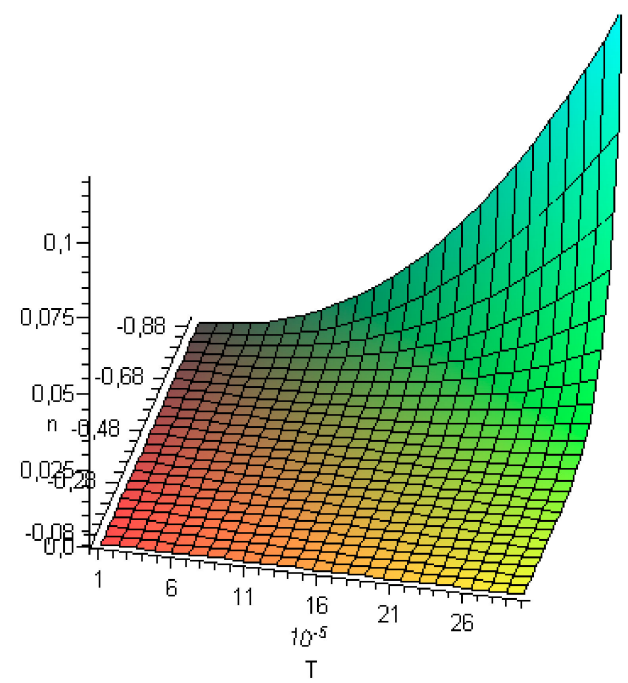

(b) $l=2, \alpha=5 \times 10^{4}$

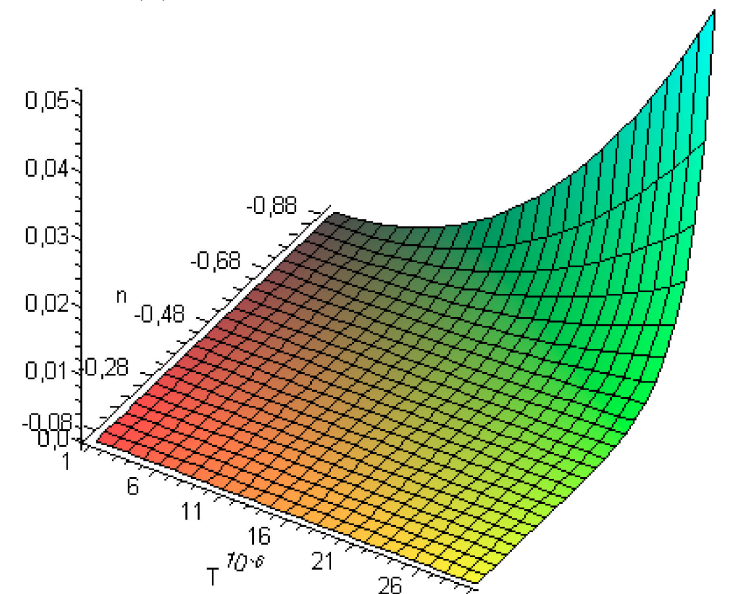

(c) $l=2, \alpha=2 \times 10^{3}$

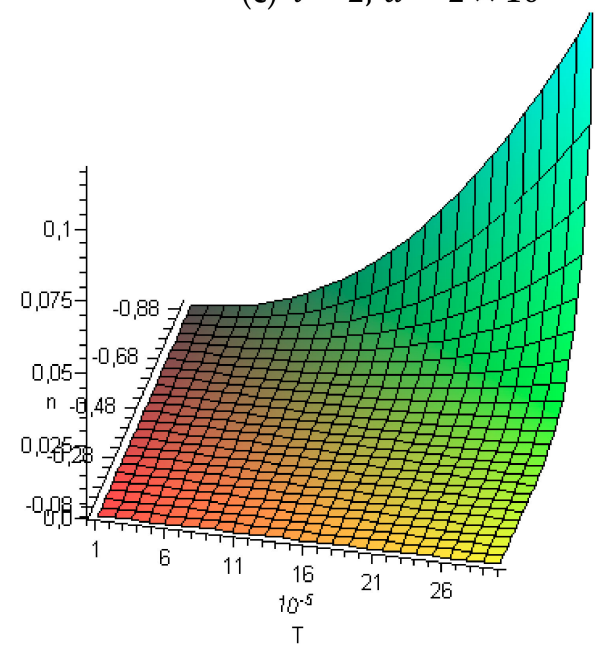

(d) $l=3, \alpha=2 \times 10^{3}$

Figure 2. Dependence of resonance amplitude $a^{*}$ from parameter $v$ and $\alpha$ : (a) $l=1, \alpha=10^{4}$, (b) $l=2, \alpha=5 \times 10^{4}$, (c) $l=2, \alpha=2 \times 10^{3}$, (d) $l=3, \alpha=2 \times 10^{3}$.

Therefore, if at the initial or at some other point in time the amplitude of oscillations takes a value greater than $a^{*}$, and the elastic body is affected only by dissipative forces and impulse periodic perturbation, the period of which satisfies the condition 
$\tau \approx \frac{2 \Pi}{\alpha\left(a^{*}\right)^{\frac{v}{2}}}\left(\frac{l}{\Pi_{x}}\right)^{1+\frac{v}{2}}$, then the elastic body will have resonant oscillations. They, as follows from the above and differential Equation (12) are described by the dependence:

$$
\begin{aligned}
& \frac{d a}{d t}=\mu A_{1}(a)-\frac{\mu}{4 \omega(a) \rho P^{2} \pi} \int_{0}^{2 \pi} \int_{0}^{l}\left\{\sum_{s=1} \cos \theta \delta\left(\frac{\theta}{\mu}-\frac{2(s-1) \pi}{\mu}\right) G_{s}(a, x, \gamma+\theta) \sum_{j=1} X_{j}\left(x_{s}\right) X_{j}(x)\right\} X(x) s a(1, v+1, \gamma+\theta) d x d \theta \\
& \frac{d \gamma}{d t}=\frac{d \omega(a)}{d a} \mid a=a *\left(a-a^{*}\right)+\mu B_{1}(a)-\frac{\mu(\nu+2)}{8 a \omega(a) \rho P^{2} \pi} \int_{0}^{2 \pi} \int_{0}^{l}\left\{\sum_{s=1} \cos \theta \delta\left(\frac{\theta}{\mu}-\frac{2(s-1) \pi}{\mu}\right) G_{s}(a, x, \gamma+\theta) \sum_{j=1} X_{j}\left(x_{s}\right) X_{j}(x)\right\} X(x) c a(v+1,1, \gamma+\theta) d x d \theta .
\end{aligned}
$$

The properties of the $\delta$ function allow relation in the Equation (14) to be somewhat simplified and presented as

$$
\begin{aligned}
& \frac{d a}{d t}=\mu A_{1}(a)-\frac{\mu}{4 \omega(a) \rho P^{2} \pi} \int_{0}^{l}\left\{\sum_{s=1} s a\left(1, v+1, \frac{\Pi}{\pi}(\gamma+2(s-1) \pi)\right) G_{s}\left(a, x, \frac{\Pi}{\pi}(\gamma+2(s-1) \pi)\right) \sum_{j=1} X_{j}\left(x_{s}\right) X_{j}(x)\right\} X(x) d x \\
& \frac{d \gamma}{d t}=\frac{d \omega(a)}{d a} \mid a=a * \\
& \left.\mid a-a^{*}\right)+\mu B_{1}(a)-\frac{\mu(v+2)}{8 a \omega(a) \rho P^{2} \pi} \int_{0}^{l}\left\{\sum_{s=1} c a\left(1, v+1, \frac{\Pi}{\pi}(\gamma+2(s-1) \pi)\right) G_{s}\left(a, x, \frac{\Pi}{\pi}(\gamma+2(s-1) \pi)\right) \sum_{j=1} X_{j}\left(x_{s}\right) X_{j}(x)\right\} X(x) d x
\end{aligned}
$$

In particular, if the momentum perturbations are constant, then the nonresonant relations in the Equation (13) are transformed into the form:

$$
\frac{d a}{d t}=\mu A_{1}(a), \frac{d \psi}{d t}=\omega(a)+\mu B_{1}(a),
$$

Because $s a(1, v+1, \psi) \int_{0}^{2 \Pi}\left\{\begin{array}{l}s a(1, v+1, \psi) \\ c a(v+1,1, \psi)\end{array}\right\} d \psi=0$. This confirms the known result [22]: for the first approximation of the asymptotic solution, the small periodic forces in the nonresonant case do not affect the course of the dynamic process. Much more important results follow for the resonant case of the action of the specified pulse perturbation. We present only the final results (Figure 3), which describe the transition of the system through the main resonance:

$$
\begin{gathered}
\frac{d a}{d t}=\mu A_{1}(a)-\frac{\mu X_{0}}{4 \omega(a) \rho P \pi} s a\left(1, v+1, \frac{\Pi}{\pi} \gamma\right) \sum_{s=1} X_{1}\left(x_{s}\right), \\
\frac{d \gamma}{d t}=\left.\frac{d \omega(a)}{d a}\right|_{a=a *}\left(a-a^{*}\right)-\mu B_{1}(a)-\frac{\mu(v+2) X_{0}}{8 a \omega(a) \rho P \pi} c a\left(v+1,1, \frac{\Pi}{\pi} \gamma\right) \sum_{s=1} X_{1}\left(x_{s}\right) .,
\end{gathered}
$$

Below, in Figure 3 for this case, it shows the change in the amplitude of oscillations of the elastic body during the transition through resonance, provided $f\left(u, u_{t}, u_{x}, u_{x x}\right)=$ $\beta_{1}\left(u_{t}\right)+\beta_{2}\left(u_{x}\right)^{2} u_{x x}$ and boundary condition in the Equation (4) $-A_{1}(a)=\bar{\beta}_{1} a, B_{1}(a)=$ $\bar{\beta}_{2} a,{ }^{2-\frac{v}{2}}, \beta_{1}, \beta_{2}, \bar{\beta}_{1}, \bar{\beta}_{2}$ it is a known steel. 


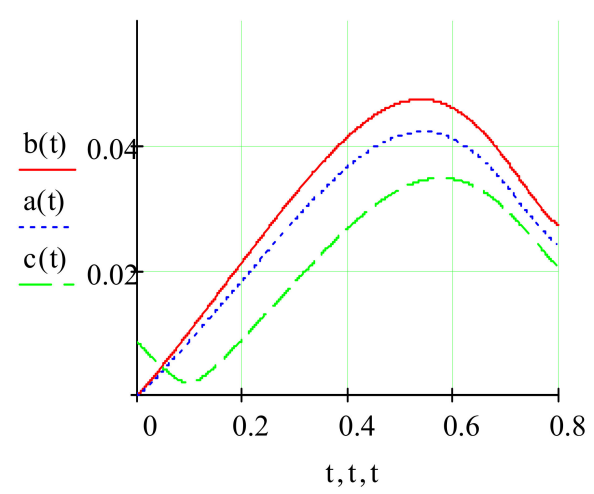

$l=2, \alpha=10^{4}, \tau=0.0004, v=-0.1, a^{*}=2.1524 \times 10^{-4}$ red,

(a) $v=-0.15, a^{*}=2.512 \times 10^{-4}$ blue, $v=-0.18, a^{*}=8.439 \times 10^{-3}$

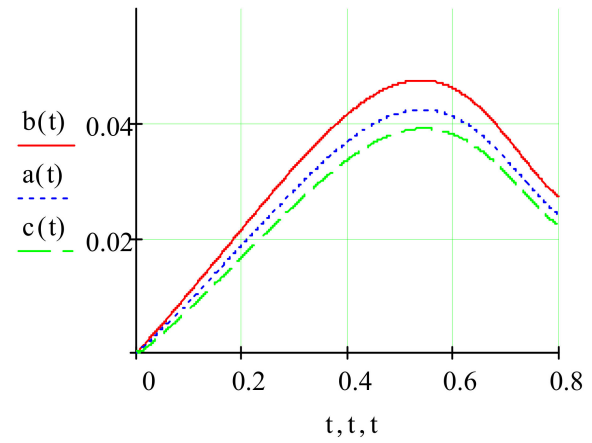

(b) $\quad l=2, \alpha=10^{4}, \tau=0.0003, v=-0.1, a^{*}=4.834 \times 10^{-7}$ red, $v=-0.15, a^{*}=6.8252 \times 10^{-7}$ blue $, v=-0.18, a^{*}=3.452 \times 10^{-4}$

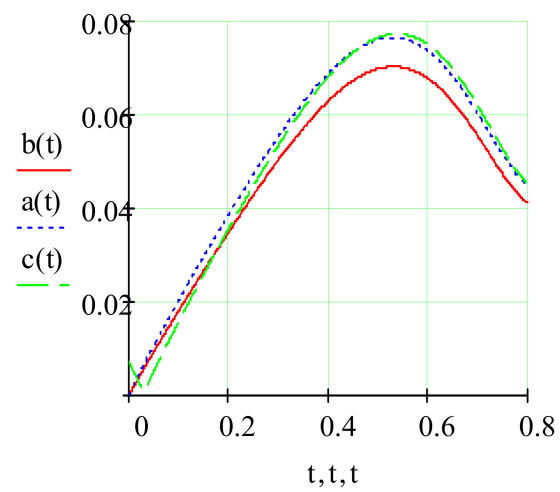

$l=2, \alpha=10^{4}, \tau=0.001, v=0.1, a^{*}=1.1648 \times 10^{-4}$ red

c) $v=0.15, a^{*}=1.649 \times 10^{-4}$ blue, $v=0.18, a^{*}=7.248 \times 10^{-3}$

Figure 3. Cont. 


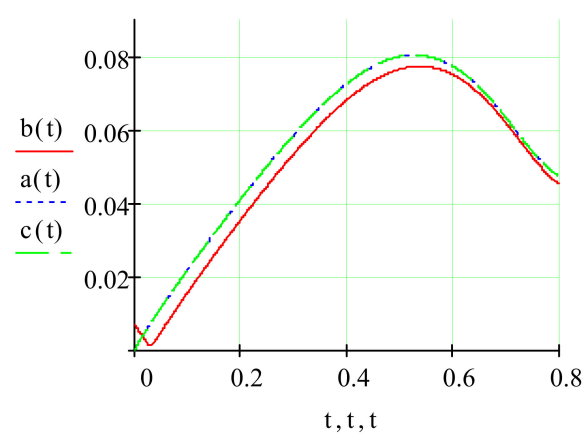

(d) $\quad l=2, \alpha=10^{4}, \tau=0.001, v=0.18, a^{*}=7.248 \times 10^{-3} \mathrm{red}$,

$\tau=0.002, v=0.18, a^{*}=3.276 \times 10^{-6}$ blue $\tau=0.003, v=0.18, a^{*}=3.621 \times 10^{-8}$

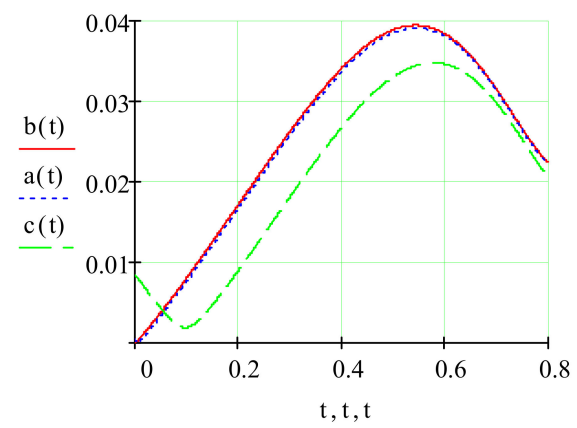

(e)

$l=2, \alpha=10^{4}, \tau=0.0002, v=-0.18, a^{*}=3.815 \times 10^{-6} \mathrm{red}$,

$\tau=0.0003, v=-0.18, a^{*}=3.452 \times 10^{-4}$ blue $, \tau=0.0004 v=-0.18, a^{*}=8.439 \times 10^{-3} \mathrm{gr}$

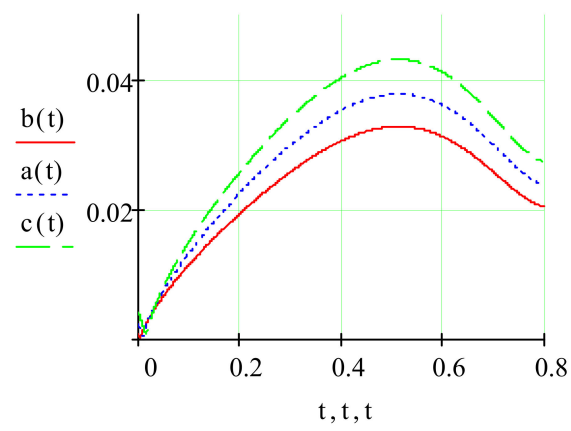

(f) $l=2, \alpha=8 \times 10^{4}, \tau=0.001, v=0.7, a^{*}=8.62 \times 10^{-4} \mathrm{red}$,

(f) $\tau=0.001, v=0.8, a^{*}=2.128 \times 10^{-3}$ blue, $\tau=0.001 v=0.9 a^{*}=4.292 \times 10^{-3}$ green

Figure 3. Change in the amplitude of longitudinal oscillations of a nonlinear elastic body at different periods of external periodic perturbation and different values of the parameter $v$ :

(a) $l=2, \alpha=10^{4}, \tau=0.0004, v=-0.1, a^{*}=2.1524 \times 10^{-4}$ red,

(a) $v=-0.15, a^{*}=2.512 \times 10^{-4}$ blue, $v=-0.18, a^{*}=8.439 \times 10^{-3}$;

$l=2, \alpha=10^{4}, \tau=0.0003, v=-0.1, a^{*}=4.834 \times 10^{-7}$ red,

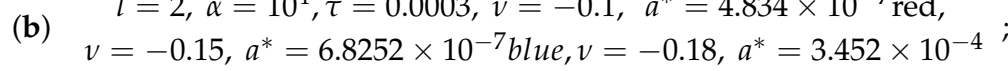

$l=2, \alpha=10^{4}, \tau=0.001, v=0.1, a^{*}=1.1648 \times 10^{-4} \mathrm{red}$,

(c) $v=0.15, a^{*}=1.649 \times 10^{-4}$ blue, $v=0.18, a^{*}=7.248 \times 10^{-3} ;$

(d) $\quad l=2, \alpha=10^{4}, \tau=0.001, v=0.18, a^{*}=7.248 \times 10^{-3}$ red,

$\tau=0.002, v=0.18, a^{*}=3.276 \times 10^{-6}$ blue $, \tau=0.003, v=0.18, a^{*}=3.621 \times 10^{-8}$ ，

$l=2, \alpha=10^{4}, \tau=0.0002, v=-0.18, a^{*}=3.815 \times 10^{-6}$ red,

(e) $\tau=0.0003, v=-0.18, a^{*}=3.452 \times 10^{-4}$ blue, $\tau=0.0004 v=-0.18, a^{*}=8.439 \times 10^{-3}$ green $;$

(f) $\quad l=2, \alpha=8 \times 10^{4}, \tau=0.001, v=0.7, a^{*}=8.62 \times 10^{-4}$ red,

$\tau=0.001, v=0.8, a^{*}=2.128 \times 10^{-3}$ blue, $\tau=0.001 v=0.9 a^{*}=4.292 \times 10^{-3}$ green 
The presented graphical dependencies show:

- for the case $v<0$ a smaller value of the specified parameter corresponds to a larger value of the resonant amplitude and at the same time the amplitude of the passage through the resonance is smaller;

- for the case $v>0$ its larger value corresponds to a larger value of the resonant amplitude and at the same time the amplitude of the resonance is larger;

- for the case $v=$ const $>0$ a smaller value of the pulse perturbation frequency corresponds to a smaller value of the resonant amplitude and a larger value of the amplitude of the passage through the resonance.

\section{Conclusions}

The method of research of influence of pulse perturbation on longitudinal oscillations of essentially nonlinear elastic bodies is developed in this work. It shows the fundamental difference between the studied process in linear (quasilinear) models of elastic bodies and those that are significantly nonlinear. If, for the former, the natural frequency does not depend on the amplitude, then for the substantially nonlinear it depends. In that case, there is a fundamental difference with the reaction of the body with nonlinearelastic characteristics to periodic perturbations: resonant phenomena in the latter can exist provided that the amplitude of oscillations approaches the value at which the natural frequency becomes equal to the amplitude of the external periodic perturbation. In addition, if the amplitude of oscillations of the body due to the perturbation of motion takes slightly smaller values than the amplitude at which resonance is possible, then the dissipative forces actually existing in the body cause a decrease in the amplitude and resonance, in which case, it will not be observed. In the case when the perturbation of motion causes greater than the amplitude of the resonance, the amplitude of oscillations of the body, the above dissipative forces lead to a decrease in the amplitude to a value close to resonance and resonance occurs in the elastic body.

The main results are shown:

- for a constant value of periodic pulsed perturbation, the amplitude of the passage through the main resonance for the rigid elastic properties of bodies $(-1<v<0)$ for larger values of the pulse perturbation frequency takes larger values and vice versa for the soft elastic properties of bodies $(v>0)$ a smaller value of the pulse perturbation frequency corresponds to a larger value of the amplitude of the passage through the resonance;

- the amplitude of the passage through the resonance depends on the point of application of the pulse perturbation and boundary conditions. In the case of an elastic body with fixed ends, the maximum value of the amplitude of passage through resonance will be for the case of impulse perturbation on the middle of the elastic body, and for an elastic body with free ends-for the case of impulse perturbation on the end of the elastic body.

Simultaneously, the results obtained in the work follow practical recommendations for avoiding resonant phenomena in nonlinearly elastic bodies that perform longitudinal oscillations and are subject to pulsed action is the frequency of pulsed action $\Omega$ should not be equal $\frac{\pi \omega(a)}{\Pi}$.

The reliability of the obtained results is confirmed by obtaining in the extreme case $\left(v=0, g_{s}\left(u, u_{t}, u_{x}, u_{x x}\right) \equiv 0\right)$ is known from literary sources.

Practical application of the obtained results: the results obtained in this work can be the basis for selecting the parameters of the elements of protective and other structures that are subject to instantaneous action, as well as, bulk media during the process of their vibro-transportation, and separation.

Author Contributions: All authors contributed equitably. All authors have read and agreed to the published version of the manuscript. 
Funding: This research was funded by the Slovakia Research and Development Agency under the contract no. APVV-19-0581 and by the Faculty of Management of Comenius University in Bratislava, Slovakia.

Institutional Review Board Statement: Not applicable.

Informed Consent Statement: Not applicable.

Data Availability Statement: Not applicable.

Conflicts of Interest: The authors declare no conflict of interest.

\section{References}

1. Pisarenko, G.S.; Kvitka, O.L.; Umansky, E.S. Resistance of Materials; Higher School: Kyiv, Ukraine, 2004; 655p, ISBN 966-642-056-2.

2. Lott, D.A.; Antman, S.S.; Szymczak, W.G. The Quasilinear Wave Equation for Antiplane Shearing of Nonlinearly Elastic Bodies. J. Comput. Phys. 2001, 171, 201-226. [CrossRef]

3. Habeck, D.; Schuricht, F. Contact between nonlinearly elastic bodies. Proc. R Soc. Edinb. Sect. A-Math. 2006, 136, 1239-1266. [CrossRef]

4. Antman, S.; Shvartsman, M. The Shrink-Fit Problem for Aeolotropic Nonlinearly Elastic Bodies. J. Elast. 1995, 37, 157-166. [CrossRef]

5. Annin, B.D.; Bondar, V.D. Antiplane Strain in a Nonlinearly Elastic Incompressible Body. J. Appl. Mech. Tech. Phys. 2006, 47, 849-856. [CrossRef]

6. Warne, P.G.; Polignone, D.A. Solutions for an infinite compressible nonlinearly elastic body under a line load. Q. Appl. Math. 1996, 54, 317-326. [CrossRef]

7. Kryvinska, N.; Bickel, L. Scenario-Based Analysis of IT Enterprises Servitization as a Part of Digital Transformation of Modern Economy. Appl. Sci. 2020, 10, 1076. [CrossRef]

8. Tkachenko, R.; Izonin, I.; Kryvinska, N.; Dronyuk, I.; Zub, K. An Approach towards Increasing Prediction Accuracy for the Recovery of Missing IoT Data based on the GRNN-SGTM Ensemble. Sensors 2020, 20, 2625. [CrossRef] [PubMed]

9. Cveticanin, L. Strong Nonlinear Oscillator-Analytical Solutions. In Mathematical Engineering; Springer International Publishing AG: New York, NY, USA, 2018; 317p. [CrossRef]

10. Cveticanin, L. Period of vibration of axially vibrating truly nonlinear rod. J. Sound Vib. 2016, 374, 199-210. [CrossRef]

11. Cveticanin, L.; Pogány, T. Oscillator with a Sum of Noninteger-Order Nonlinearities. J. Appl. Math. 2012, 2012, 1-20. [CrossRef]

12. Gendelman, O.; Vakakis, A.F. Transitions from localization to nonlocalization in strongly nonlinear damped oscillators. Chaos, Solitons Fractals 2000, 11, 1535-1542. [CrossRef]

13. Sokil, B.I.; Barvinskii, A.F. An asymptotic expansion for a class of nonlinear differential equations. Ukr. Math. J. 1981, 32, 465-468. [CrossRef]

14. Sokil, B.I. Construction of asymptotic solutions of certain boundary-value problems for the nonautonomous wave equation. J. Math. Sci. 1999, 96, 2878-2882. [CrossRef]

15. Pukach, P.Y. Qualitative Methods for the Investigation of a Mathematical Model of Nonlinear Vibrations of a Conveyer Belt. J. Math. Sci. 2014, 198, 31-38. [CrossRef]

16. Antman, S.S.; Zhong-Heng, G. Large shearing oscillations of incompressible nonlinearly elastic bodies. J. Elast. 1984, 14, $249-262$. [CrossRef]

17. Myshkis, A.D.; Filimonov, A.M. Periodic oscillations in nonlinear one-dimensional continuous media. In Proceedings of the IX International Conference on Nonlinear Oscillations, Kyiv, Ukraine, 30 August-6 September 1981; Part 1, Analytical methods. Naukova Dumka: Kyiv, Ukraine, 1984; pp. 274-276.

18. Erbay, H.A.; Erbay, S.; Erkip, A. The Cauchy problem for a class of two-dimensional nonlocal nonlinear wave equations governing anti-plane shear motions in elastic materials. Nonlinearity 2011, 24, 1347-1359. [CrossRef]

19. Räty, R.; Von Boehm, J.; Ranta, M. Non-linearly Forced Vibrations of Elastic Systems Carrying Rigid Bodies. J. Sound Vib. 1994, 169, 71-87. [CrossRef]

20. Delta Function. Mathematics. Available online: https:/ / mathworld.wolfram.com/DeltaFunction.html (accessed on 20 July 2020).

21. Zelenina, A.; Zubov, L.M. One-dimensional deformations of nonlinearly elastic micropolar bodies. Mech. Solids 2010, 45, 575-582. [CrossRef]

22. Liu, X.Q.; Ni, X.H.; Ma, Y.C. Elastic-plastic analysis of nonlinearly elastic structure in the space bars pinned on a rigid body. In Proceedings of the 4th International Symposium on Test and Measurement, Shanghai, China, 1-3 June 2001; Chinese Society of Modern Technical Equipments: Shanghai, China, 2001; Volume 1-2, pp. 1084-1086.

23. Cattani, C.; Rushchitskii, Y.Y. The subharmonic resonance and second harmonic of a plane wave in nonlinearly elastic bodies. Int. Appl. Mech. 2003, 39, 93-98. [CrossRef]

24. Andrukhiv, A.; Sokil, M.; Fedushko, S.; Syerov, Y.; Kalambet, Y.; Peracek, T. Methodology for Increasing the Efficiency of Dynamic Process Calculations in Elastic Elements of Complex Engineering Constructions. Electronics 2021, 10, 40. [CrossRef] 
25. Ni, X.H.; Liu, X.Q.; Lu, X.B. The stress-strain analysis of the nonlinearly elastic structure of the bars pinned to a turntable rigid body. In Proceedings of the 4th International Symposium on Test and Measurement, Shanghai, China, 1-3 June 2001; Chinese Society of Modern Technical Equipments: Shanghai, China, 2001; Volume 1-2, pp. 1039-1040. 